Kihm, Pascal; Peschel, Markus

\title{
doing AGENCY - der Transfer von AGENCY-Elementen in Lernwerkstätten am Beispiel des Grundschullabors für Offenes Experimentieren
}

Tänzer, Sandra [Hrsg.]; Godau, Marc [Hrsg.]; Berger, Marcus [Hrsg.]; Mannhaupt, Gerd [Hrsg.]: Perspektiven auf Hochschullernwerkstätten. Wechselspiele zwischen Individuum, Gemeinschaft, Ding und Raum. Bad Heilbrunn : Verlag Julius Klinkhardt 2019, S. 184-187. - (Lernen und Studieren in Lernwerkstätten)

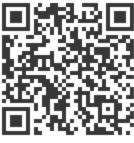

Quellenangabe/ Reference:

Kihm, Pascal; Peschel, Markus: doing AGENCY - der Transfer von AGENCY-Elementen in Lernwerkstätten am Beispiel des Grundschullabors für Offenes Experimentieren - In: Tänzer, Sandra [Hrsg.]; Godau, Marc [Hrsg.]; Berger, Marcus [Hrsg.]; Mannhaupt, Gerd [Hrsg.]: Perspektiven auf Hochschullernwerkstätten. Wechselspiele zwischen Individuum, Gemeinschaft, Ding und Raum. Bad Heilbrunn : Verlag Julius Klinkhardt 2019, S. 184-187 - URN: urn:nbn:de:0111-pedocs-201981 - DOI: 10.25656/01:20198

https://nbn-resolving.org/urn:nbn:de:0111-pedocs-201981

https://doi.org/10.25656/01:20198

in Kooperation mit / in cooperation with:

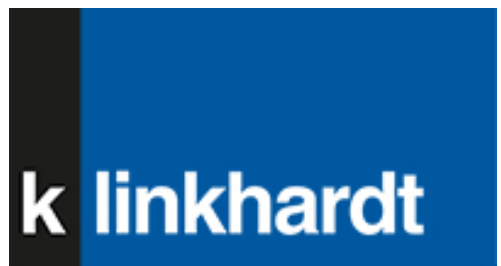

http://www.klinkhardt.de

\section{Nutzungsbedingungen}

Dieses Dokument steht unter folgender Creative Commons-Lizenz: http://creativecommons.org/licenses/by-nc-sa/4.0/deed.de - Sie dürfen das Werk bzw. den Inhalt unter folgenden Bedingungen vervielfältigen, verbreiten und öffentlich zugänglich machen sowie Abwandlungen und Bearbeitungen des Werkes bzw. Inhaltes anfertigen: Sie müssen den Namen des Autors/Rechteinhabers in der von ihm festgelegten Weise nennen. Dieses Werk bzw. der Inhalt darf nicht für kommerzielle Žwecke verwendet werden. Die neu entstandenen Werke bzw. Inhalte dürfen nur unter Verwendung von Lizenzbedingungen weitergegeben werden, die mit denen dieses Lizenzvertrages identisch oder vergleichbar sind.

Mit der Verwendung dieses Dokuments erkennen Sie die Nutzungsbedingungen an.

\section{Terms of use}

This document is published under following Creative Commons-License: http://creativecommons.org/licenses/by-nc-sa/4.0/deed.en - You may copy, distribute and transmit, adapt or exhibit the work in the public and alter, transform or change this work as long as you attribute the work in the manner specified by the author or licensor. You are not allowed to make commercial use of the work. If you alter, transform, or change this work in any way, you may distribute the resulting work only under this or a comparable license.

By using this particular document, you accept the above-stated conditions of use.

\section{Kontakt / Contact:}

peDOCS

DIPF | Leibniz-Institut für Bildungsforschung und Bildungsinformation Informationszentrum (IZ) Bildung

E-Mail: pedocs@dipf.de

Internet: www.pedocs.de

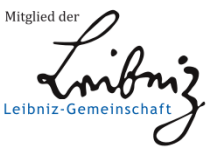


UNIVERSITÄT

Bislang wird der Begriff AGENCY vornehmlich im Kontext sozial- und kulturwissenschaftlicher Fragen konzeptualisiert:

$>$ Welche Möglichkeiten der Einflussnahme auf das eigene Handeln haben Akteurinnen und Akteure im Rahmen der strukturellen, individuellen und sozialen Bedingungen einer Situation (Handlungsfähigkeit)?

$>$ In welchem Maße können Individuen in einer Situation unabhängig von verdeckten oder offen zu Tage tretenden Strukturen und Machtverhältnissen - d.h. selbstbestimmt - agieren (Selbstbestimmung)?

$>$ Wie wird diese Handlungsfähigkeit und Selbstbestimmung einer Akteurin bzw. eines Akteurs in der jeweiligen Situation zwischen den Beteiligten ausgehandelt?

Das AGENCY-Konzept wird auf das Experimentieren von Kindern in der Lernwerkstatt GoFEx (Grundschullabor für Offenes Experimentieren) und auf die dabei stattfindende pädagogisch-didaktische Begleitung (Lernwerkstattbegleitung) transferiert, um sachunterrichtliche Näherungen an Experimentieren in Lernwerkstätten zu erforschen.

Aufgaben fungieren als Vermittler zwischen Kind, Experimentier-/Lernprozess, Lehrperson und Sache. Vor allem drei Öffnungsdimensionen (methodisch, inhaltlich, organisatorisch) werden in der Konzeption von Aufgaben adressiert. Ferner zeigt sich die soziale und persönliche Offenheit insbesondere bei der pädagogisch-didaktischen Lern(werkstatt)begleitung über das (Sach-)Lehren. Prozesse der Aushandlung von AGENCY beim Experimentieren sollen erforscht werden.

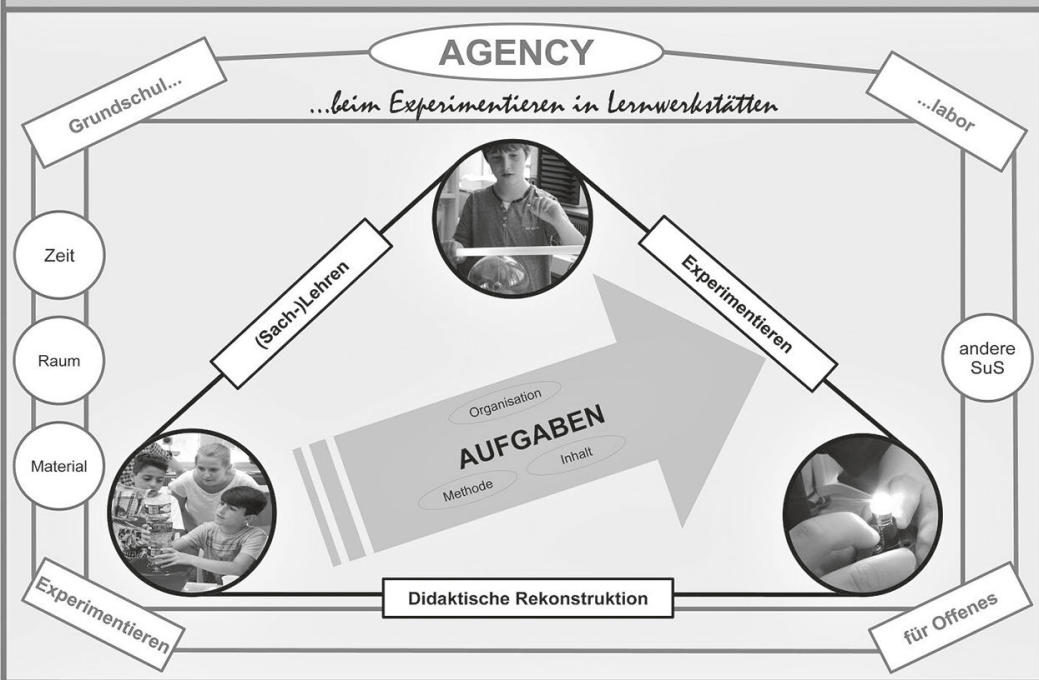

Forschungsinteressen:

Aushandlung von Selbstbestimmung (doing AGENCY) beim Experimentieren in der Lernwerkstatt GoFEX

$>$ Welche Rolle spielen dabei die differenten Aufgabenformulierungen auf den modularen Öffnungsgraden des GoFEX?

- Welche Rolle spielt dabei der Einsatz von Aufgaben an Schülertagen im GoFEX sowie der Umgang mit diesen Aufgaben durch Lehrkräfte und das "GOFEX-Team" (pädagogisch-didaktische Lernbegleitung, Interventionen usw.)?

$>$ Wie wird fachliche Handlungs- und Entscheidungsmacht (Autorität) im GofEX ausgehandelt:

Wer sagt wann und wie, was "richtig" ist? Wie wird mit "Aufgaben-Grenzen" umgegangen?

> Wie wird AGENCY - verstanden als Selbstbestimmung - beim Experimentieren in Lernwerkstätten ausgehandelt?

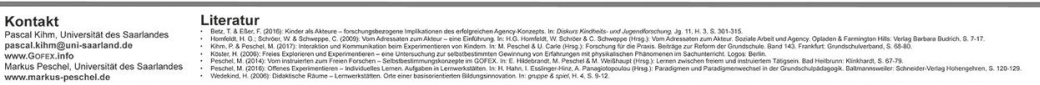




\section{doing AGENCY - der Transfer von AGENCY-Elementen in Lernwerkstätten am Beispiel des Grundschullabors für Offenes Experimentieren}

„Aushandlung von Selbstbestimmung“ ist ein Element bei der Arbeit im GoFEX (Grundschullabor für Offenes Experimentieren; vgl. Peschel 2014), einer Hochschullernwerkstatt an der Universität des Saarlandes, in der Kompetenzen für (Offenes) Experimentieren entwickelt werden können. Selbstkonstruktionsprozesse der Lernenden und verschiedene Möglichkeiten naturwissenschaftlicher Erkenntnisgewinnung - unter Fokussierung von Explorationen, Beobachtungen, Wiederholungen, Variationen, Austausch und Reflexionen - stehen im Zentrum des Experimentierens im Gofex (vgl. Peschel 2016).

Für das Dissertationsprojekt doing AGENCY (Pascal Kihm) werden Elemente des AGENCY-Ansatzes adaptiert, um Selbstbestimmung in der Lehr-Lern-Umgebung Gofex (vgl. Peschel 2014) spezifischer zu erforschen. In den Sozialwissenschaften wird der AGENCY-Ansatz herangezogen, um „Fragen der Herstellung, Absicherung oder Ermöglichung von Handlungsfähigkeit" (Raitelhuber 2012, 122) in sozialen Situationen zu untersuchen. Selbstbestimmung wird in diesem Ansatz nicht allein als individualpsychologische Eigenschaft konzeptualisiert, sondern ist jeweils neu in einer Situation zwischen den beteiligten Akteur*innen auszuhandeln (vgl. ebd.). In der Sachunterrichtsdidaktik ist AGENCY u.E. bislang wenig etabliert und kaum in entsprechende Forschungen eingeflossen.

Ziel der Erforschung der AGENCY-Komponente im GofEx ist es, zu verstehen, wie Selbstbestimmung beim Experimentieren zwischen den beteiligten Akteur*innen (Lernbegleitung, Lehrkräfte, Mitschüler*innen) und dem Phänomen bzw. der Sache ausgehandelt wird. Durch ein Verständnis der Aspekte, die für einen selbstbestimmten Lehr-Lern- bzw. Experimentierprozess in einer Klasse eine Rolle spielen, kann daraufhin ggf. ein erweitertes Rollenverständnis zwischen Lehrenden und Lernenden etabliert und das Lernen bzw. Experimentieren von Kindern anders begleitet werden (vgl. Peschel 2016, 122f.; Wedekind 2016, 207f.). 
Konzepte und Begriffe um „Offenes Experimentieren“ werden u.E. vielfach undifferenziert verwendet (vgl. Peschel 2009, 268f.; Priemer 2011, 322f.). ${ }^{1}$ Die konzeptionelle und begriffliche Vielfalt in den Untersuchungen erlaubt es, „fast jede Unterrichtssituation nahezu völlig beliebig als offen oder als geschlossen zu werten" (Peschel, F. 2012, 48).

Zudem bleibt das Verständnis von Offenheit in bisherigen Untersuchungen meist beschränkt (vgl. ebd.). So wird z.B. u.E. nicht genügend berücksichtigt, dass die verschiedenen Öffnungsaspekte des Offenen Experimentierens im Sachunterricht letztlich sozial ausgehandelt werden (vgl. Lipowsky 2002, 142ff.; Köster 2006, 155ff.; Peschel 2014, 77f.). Mittels AGENCY kann es jedoch ermöglicht werden, die bisherigen Forschungsansätze um die soziale und persönliche Offenheitsdimensionen nach Falko Peschel (2012) zu erweitern.

In ersten Vorarbeiten konnten Kihm \& Peschel (2017) sowie Diener \& Peschel (2019 i.V.) durch Beobachtungsverfahren (Videographie bzw. teilnehmende Beobachtung mit Feldnotizen) rekonstruieren, dass die Aushandlung von Selbstbestimmung beim Experimentieren durch (1) die Offenheit bzw. Geschlossenheit einer Aufgabe, (2) die Gestaltung der Lernumgebung sowie (3) die Zugänglichkeit und Anordnung von Materialien grundgelegt wird. Diese drei Aspekte zeigen deutliche Auswirkungen auf die Handlungs- und Wahlmöglichkeiten im Hinblick auf Inhalte und Methoden. Die mittels verschiedener Aufgabenformate grundgelegte Aushandlung von Selbstbestimmung wird in der sozialen Interaktion zwischen den Lernenden bzw. mit der Lehrperson fortgeführt und ausgeweitet. In Zukunft soll dabei u.a. die Frage fokussiert werden, wie sich Interventionen und Lernbegleitungsmaßnahmen von Lehrer*innen auf die in Experimentieraufgaben adressierte organisatorische, methodische bzw. inhaltliche (ggf. auch soziale) Offenheit auswirken.

Das Dissertationsvorhaben doing AGENCY will somit einen Beitrag zur bislang eher defizitären Forschungslage zu Öffnungsaspekten und -dimensionen beim Experimentieren im Sachunterricht leisten. Dies könnte das Verständnis darüber verändern, wie Lernwerkstätten mit Selbstbestimmung und Offenheit umgehen. Schließlich nehmen soziale Prozesse und Aushandlungen in der Lernwerkstattarbeit eine besondere Rolle ein (vgl. Wedekind 2016).

1 In vielen Untersuchungen in der Primarstufe, die „offene“ Stichproben ausweisen (z.B. Hardy et al. 2006; Möller 2016), haben Grundschüler*innen (fast) ausschließlich organisatorische Entscheidungsmöglichkeiten (bzgl. Ort, Zeit, Sozialform). Dahingegen beschreiben Kihm und Peschel (2017) oder Köster (2006) in ihren Untersuchungen auch eine methodische (Lern- und Lösungswege, Lernziele) und ggf. sogar inhaltliche Öffnung (Lerninhalt) des Experimentierens der Schüler*innen in einer Lernwerkstatt bzw. im Sachunterricht. Insofern stecken hinter der Einschätzung der „Offenheit“ in bisherigen Ansätzen zur Erforschung „Offenen Experimentierens“ im Grundschulbereich durchaus sehr unterschiedliche Situationen mit differenten Handlungs- und Entscheidungsmöglichkeiten von Schüler*innen. 


\section{Literatur}

Diener, Jenny \& Peschel, Markus (2019 i.V.): Lehrerhandeln im Grundschullabor für Offenes Experimentieren (Gofex). In: Peschel, Markus (Hrsg.): Praxisforschung Sachunterricht - Beispiele für gute sachunterrichtliche Praxis. Kinder.Sachen. Welten. Dimensionen des Sachunterrichts. Baltmannsweiler: Schneider Verlag Hohengehren.

Hardy, Ilonca; Jonen, Angela; Möller, Kornelia; Stern, Elsbeth (2006): Effects of instructional support within constructivist learning environments for elementary school students' understanding of "floating and sinking”. In: Journal of Educational Psychology, 98 Jg., H. 2, 307-326.

Kihm, Pascal \& Peschel, Markus (2017): Interaktion und Kommunikation beim Experimentieren von Kindern - Eine Untersuchung über interaktions- und kommunikationsförderliche Aufgabenformate. In: Peschel, Markus \& Carle, Ursula (Hrsg.): Forschung für die Praxis, Beiträge zur Reform der Grundschule. Frankfurt am Main: Grundschulverband, 66-80.

Köster, Hilde (2006): Freies Explorieren und Experimentieren - eine Untersuchung zur selbstbestimmten Gewinnung von Erfahrungen mit physikalischen Phänomenen im Sachunterricht. Berlin: Logos Berlin.

Lipowsky, Frank (2002): Zur Qualität offener Lernsituationen im Spiegel empirischer Forschungen. Auf die Mikroebene kommt es an. In: Drews, Ursula \& Wallrabenstein, Wulf (Hrsg.): Freiarbeit in der Grundschule. Offener Unterricht in Theorie, Forschung und Praxis, Beiträge zur Reform der Grundschule. Frankfurt am Main: Grundschulverband, 126-159.

Möller, Kornelia (2016): Bedingungen und Effekte qualitätsvollen Unterrichts - ein Beitrag aus fachdidaktischer Perspektive. In: McElvany, Nele; Bos, Wilfried; Holtappels, Heinz Günter; Gebauer, Miriam M. \& Schwabe, Franziska (Hrsg.): Bedingungen und Effekte guten Unterrichts, Dortmunder Symposium der Empirischen Bildungsforschung. Münster; New York: Waxmann, 43-64.

Peschel, Falko (2012): Offener Unterricht - Idee, Realität, Perspektive und ein praxiserprobtes Konzept in der Evaluation. Baltmannsweiler: Schneider Verlag Hohengehren.

Peschel, Markus (2009): Der Begriff der Offenheit beim Offenen Experimentieren. In: Höttecke, D. (Hrsg.): Chemie- und Physikdidaktik für die Lehramtsausbildung. Münster: LIT, 268-270.

Peschel, Markus (2014): Vom instruierten zum Freien Forschen - Selbstbestimmungskonzepte im GOFEX. In: Hildebrandt, Elke; Peschel, Markus \& Weißhaupt, Mark (Hrsg.): Lernen zwischen freiem und instruiertem Tätigsein. Lernen und Studieren in Lernwerkstätten - Impulse für Theorie und Praxis einer innovativen Lehrerbildung. Bad Heilbrunn: Klinkhardt, 67-79.

Peschel, Markus (2016): Offenes Experimentieren - Individuelles Lernen. Aufgaben in Lernwerkstätten. In: Hahn, Heike; Esslinger-Hinz, Ilona \& Panagiotopoulou, Argyro (Hrsg.): Paradigmen und Paradigmenwechsel in der Grundschulpädagogik. Baltmannsweiler: Schneider Verlag Hohengehren, 120-129.

Priemer, Burkhard (2011): Was ist das Offene beim offenen Experimentieren? In: Zeitschrift für Didaktik der Naturwissenschaften, 17 Jg., 315-337.

Raitelhuber, Eberhard (2012): Ein relationales Verständnis von Agency. Sozialtheoretische Überlegungen und Konsequenzen für empirische Analysen. In: Bethmann, Stephanie; Helfferich, Cornelia; Hoffmann, Heiko \& Niermann, Debora (Hrsg.): Agency: Qualitative Rekonstruktionen und gesellschaftstheoretische Bezüge von Handlungsmächtigkeit. Weinheim: Beltz Juventa, 122-154.

Wedekind, Hartmut (2016): Das Kinderforscherzentrum HELLEUM. Eine Lernwerkstatt für naturwissenschaftlich-technische Bildung in der frühen Kindheit. In: Schude, Sabrina; Bosse, Dorit \& Klusmeyer, Jens (Hrsg.): Studienwerkstätten in der Lehrerbildung. Theoriebasierte Praxislernorte an der Hochschule. Wiesbaden: Springer Fachmedien, 205-219. 